\title{
On a Slightly Different Power Law-Scaling for the Flat Universe
}

\author{
C. Sivakumar*, R. Francis \\ Department of Physics, Maharaja's College, Ernakulam, Kochi-682011, Kerala, India
}

Received 20 April 2020, accepted in final revised form 23 June 2020

\begin{abstract}
A slightly different power law-scaling fits to the picture of our 13.7 billion years old flat universe which is expanding presently at $67 \mathrm{~km} / \mathrm{s} / \mathrm{Mpc}$ with an acceleration. The model which is an attempt to retain power-law scaling in the light of the accepted facts about the universe we are living in, has a constant effective equation of state parameter as the cosmic fluid is a solution of matter, radiation and dark energy. It is successful in explaining the acceleration of universe which the normal power law fails if the present Hubble parameter is $67 \mathrm{~km} / \mathrm{s} / \mathrm{Mpc}$ and age of the universe is 13.7 billion years, and it is free from the defect of singularity.
\end{abstract}

Keywords: Hubble parameter; Power law-scaling; Equation of state parameter; Dark energy.

() 2020 JSR Publications. ISSN: 2070-0237 (Print); 2070-0245 (Online). All rights reserved.

doi: http://dx.doi.org/10.3329/jsr.v12i4.46721 J. Sci. Res. 12 (4), 569-574 (2020)

\section{Introduction}

The hot big bang model or standard cosmological model [1-3], which has maximum consistency with observations is based upon the mathematically elegant assumptions of spatial homogeneity and isotropy (cosmological principle) through the maximally spatially symmetric Friedmann-Robertson-Walker metric. This model gives us a good account of evolution of the early universe. However in this classical general relativistic model, a singularity in the past is unavoidable and is also associated with many problems like horizon problem, flatness problem, age problem, cosmological constant problem etc. [4-6]. A lot of cosmological models were proposed in the literature [7-10] which discuss these problems, all of which are based on Friedman-Robertson-Walker metric.

$$
d s^{2}=c^{2} d t^{2}-R^{2}(t)\left[\frac{d r^{2}}{\sqrt{1-k r_{1}^{2}}}+r_{1}^{2}\left(d \theta^{2}+\sin ^{2} \theta d \varphi^{2}\right)\right]
$$

Here $R(t)$ is called scale factor for the universe, which is a measure of the size of the universe and increases with expansion of the universe. $k$ is called curvature parameter, which can take three possible values: $k=+1,0,-1$ and space is positively curved, flat or negatively curved respectively. The corresponding Friedmann models are called closed, flat and open models. $\left\{t, r_{1}, \theta, \varphi\right\}$ are the commoving coordinates in spherical polar

\footnotetext{
*Corresponding author: thrisivc@yahoo.com
} 
system. We know that standard cosmology has its theoretical foundation on Friedmann [11] equations (which are solutions of Einstein's field equations with above metric) with power law scaling as a simple and elegant solution and Cosmic Microwave Background Radiation (CMBR) is the strongest piece of experimental evidence for the evolution of the universe from a denser state to the present state [12].

The brightest microwave fluctuations that have been measured by Wilkinson Microwave Anisotropy Probe (WMAP) [13] to the accuracy of 0.004 are about $1^{\circ}$ across to show that ours is a flat universe. The results of supernova cosmology project (SCP) $[14,15]$ have established the fact that universe has an accelerated expansion. These observations also point to the need of incorporating a missing energy (dark energy) density in the total energy density, which possess a negative pressure. Many candidates were proposed for this unknown dark energy [16-22] like quintessence, tracker fields, cosmological constant, vacuum energy etc. Recent measurements suggest that at present our universe is dark energy dominated and equation of state parameter $\omega$ is less than $-1 / 3$ to account for the accelerating expansion rate for the universe. Planck mission [23] and other recent measurements [24] suggest that the equation of state parameter of dark energy is -0.6 at least.

From the apparent magnitude-redshift data [23] of the distant type Ia supernovae, Hubble parameter has been fine tuned to $67 \mathrm{~km} / \mathrm{s} / \mathrm{Mpc}$. Melia et al. [25] proposed a model called $R_{h}=c t$ model, in which the authors proposed $\omega=-1$ for dark energy and $\rho+3 p=0$ to form a coasting evolution for the universe. John [26] argues that this model resembles their eternal coasting model published in [27]. However these models lacks consistency with recent observations of acceleration in expansion. The conservation condition in their models only lead to a uniform expansion. To protect recent results and also to stick on to the power law-scaling we propose a simple correction to produce a singularity-free 13.7 billion year (by) old flat universe that is expanding presently at a rate of $67 \mathrm{~km} / \mathrm{s} / \mathrm{Mpc}$ [28] with an acceleration. The plan of the paper is as follows: section 2 describes the new model, section 3 discusses the results of our model and section 4 is about the conclusions.

\section{Materials and Methods}

We use Einstein's equations for an isotropic homogeneous universe, so space-time is described by Friedmann-Robertson-Walker (FRW) metric and energy momentum tensor is that of a perfect fluid with energy density $\rho(t)$ and pressure $P(t)$ which are functions of the time coordinate $t$ that is proper time for observers moving with the fluid. The resulting equations are called Friedmann equations and for flat geometry they are given by,

$$
\begin{aligned}
& \frac{\dot{R}^{2}}{R^{2}}-\frac{8 \pi G \rho}{3}=0, \quad \frac{\dot{R}}{R}=H(t) \\
& 2 \frac{\ddot{R}}{R}+\frac{\dot{R}^{2}}{R^{2}}+\frac{8 \pi G P(t)}{c^{2}}=0
\end{aligned}
$$

Here $\rho$ is also the critical density of the cosmic fluid that has matter, radiation and dark energy. To solve Friedman equations, we need one more equation as there are three 
functions and that is the equation of state: $P=\omega \rho c^{2}$. Equation of state is nothing but the statement of how density $\rho$ and pressure $P$ of the fluid are related to each other. $\omega$ is called equation of state parameter. For instance, if the fluid is a gas of photons (radiation), $\omega=1 / 3$.

$R$ is the scaling of the universe and $H=\frac{\dot{R}}{R}$ the Hubble parameter.

$$
\ddot{R}=-\frac{4 \pi G}{3} \rho R(1+3 \omega)
$$

An initial state and cosmic acceleration then needs

$$
-1<\omega<-\frac{1}{3}
$$

If $R=\beta t^{n}, \frac{d \beta}{d t}=0, \frac{d n}{d t}=0$ as in the Friedmann model of flat universe, then $n=t H(t)$. Using the present $\mathrm{H}, n=0.94<1.0$. But for $\ddot{R}>0, n>1.0$. To stick on to the simple power law-scaling protecting the experimental evidence for acceleration of the universe and also the present Hubble parameter and the age of the universe we postulate:

$$
H(t)=\frac{A}{t+b}
$$

(The other way of doing it is of course to modify the age of the universe; we would need then a universe at least 14.593 billion years aged so that $n>1.0$.)

\section{Results and Discussion}

Integrating $\frac{\dot{R}}{R}=\frac{A}{t+b}$,

$$
\begin{aligned}
& \log _{e} R(t)-\log _{e} R(0)=A\left[\log _{e}(t+b)-\log _{e} b\right] \\
& R(t)=\frac{R(t)(t+b)^{A}}{b^{A}} \\
& R(t)=r(t+b)^{A}, r=\frac{R(0)}{b^{A}}
\end{aligned}
$$

Differentiating Eq. (6) twice, $\ddot{R}=r A(A-1)(t+b)^{A-2}$ and hence,

$$
\ddot{R}(t)=a(t+b)^{A-2}, a=r A(A-1)
$$

From Eq. (1) we get

$$
\rho(t)=\frac{3 A^{2}}{8 \pi G} \frac{1}{(t+b)^{2}}
$$

Also,

$$
P(t)=\omega \rho(t) c^{2}=\frac{3 A^{2} \omega c^{2}}{8 \pi G} \frac{1}{(t+b)^{2}}
$$

Source of gravity is:

$$
S_{G}(t)=3 P(t)+\rho(t) c^{2}=\frac{3 c^{2}}{8 \pi G}\left(\frac{A}{t+b}\right)^{2}(3 \omega+1)
$$

Substituting H, P and R in Eq. (2), 


$$
A=1-\frac{(1+3 \omega)}{(3+3 \omega)}
$$

Now substitute A in $\mathrm{H}$,

$$
b=\frac{\left[1-\frac{1+3 \omega}{3+3 \omega}\right]}{H(t)}-t
$$

From energy conservation law: $d U+P d V=0=\omega \rho d\left(R^{3}\right)+d\left(R^{3} \rho\right)$ gives,

$$
\rho(t)=D R(t)^{-3(1+\omega)}, D=\rho(0) R(0)^{3(1+\omega)}
$$

Initial state of the universe can be described as:

$$
\begin{aligned}
& R(t)=R(0) \\
& H(t)=H(0)=\frac{A}{b} \\
& \frac{d^{2} R}{d t^{2}}(t)=\frac{d^{2} R}{d t^{2}}(0)=a b^{A-2} \\
& \rho(t)=\rho(0)=\frac{3 A^{2}}{8 \pi G b^{2}} \\
& P(t)=P(0)=\frac{3 \omega c^{2} A^{2}}{8 \pi G b^{2}} \\
& S_{G}(t)=S_{G}(0)=\frac{3 A^{2} c^{2}}{8 \pi G b^{2}}(3 \omega+1)
\end{aligned}
$$

A table showing $\omega$ and $A-1$ is shown below:

Table 1. Equation of state parameter and constant A.

\begin{tabular}{cc}
\hline$\omega$ & $\mathrm{A}-1$ \\
\hline 0.333 & -0.500 \\
0.300 & -0.487 \\
0.200 & -0.444 \\
0.100 & -0.394 \\
0.000 & -0.333 \\
-0.100 & -0.259 \\
-0.200 & -0.166 \\
-0.300 & -0.048 \\
-0.333 & 0.000 \\
-0.400 & 0.111 \\
-0.500 & 0.333 \\
-0.600 & 0.666 \\
-0.666 & 1.000 \\
-0.700 & 1.222 \\
0.800 & 2.333 \\
-0.900 & 4.666 \\
-1.000 & $\infty$ \\
\hline
\end{tabular}

Table 2. Equation of state parameter and expansion rate.

\begin{tabular}{ccc}
\hline$\omega$ & $\mathrm{A}$ & $\mathrm{dR} / \mathrm{dt}$ \\
\hline$<-0.666$ & $>2$ & increases in time \\
$=-0.666$ & $=2$ & remains the same \\
$>-0.666$ & $<2$ & decreases in time \\
\hline
\end{tabular}


Table 3. Equation of state parameter versus density.

\begin{tabular}{lc}
\hline$\omega$ & $\rho(\mathrm{t})$ \\
\hline 0.333 & $\propto R^{-4}$ \\
0 & $\propto R^{-3}$ \\
-0.333 & $\propto R^{-2}$ \\
-0.600 & $\propto R^{-1.2}$ \\
-0.666 & $\propto R^{-1}$ \\
-1.000 & Constant in time \\
\hline
\end{tabular}

If $\omega$ is taken as $-0.600, R(t)=R(0)\left(\frac{t+b}{b}\right)^{A}$, $A=1.666, b=10.657 b y$ for any $t$
Table 4. Fall of Hubble parameter with epoch.

\begin{tabular}{cc}
\hline Age $t$ in by & $H_{(t)}$ in $\mathrm{km} / \mathrm{s} / \mathrm{Mpc}$ \\
\hline 0 & 152.6 \\
1 & 138.9 \\
2 & 128.1 \\
3 & 118.3 \\
4 & 110.5 \\
5 & 103.7 \\
6 & 97.8 \\
7 & 91.9 \\
8 & 87.0 \\
9 & 82.1 \\
10 & 78.2 \\
11 & 74.3 \\
12 & 71.4 \\
13 & 68.5 \\
13.7 & 67 \\
$\cdot$ & $\cdot$ \\
. &. \\
$\infty$ & 0 \\
\hline
\end{tabular}

Table 5. Cosmological parameters at turning points.

\begin{tabular}{ccccc}
\hline Age $\mathrm{t}$ in by & $H_{(t)}$ in $\mathrm{km} / \mathrm{s} / \mathrm{Mpc}$ & $\rho_{(t)}$ in $\mathrm{kg} / \mathrm{m}^{3}$ & $P_{(t)}$ in Pa & $S_{G(t)}$ in Pa \\
\hline 0 & +152.6 & $+43 \times 10^{-27}$ & $-232.2 \times 10^{-11}$ & $-309.6 \times 10^{-11}$ \\
13.7 & +67 & $8 \times 10^{-27}$ & $-43.2 \times 10^{-11}$ & $-57.6 \times 10^{-11}$ \\
$\infty$ & +0 & +0 & -0 & -0 \\
\hline
\end{tabular}

\section{Conclusion}

The model is a fluid that started off from a non-singular state of $43 \times 10^{-27} \mathrm{~kg}$ of mass per unit volume with a negative pressure of size $232.2 \times 10^{-11} \mathrm{~Pa}$ under the effect of dark energy with a speed of expansion of $152.6 \mathrm{~km} / \mathrm{s} / \mathrm{Mpc}$; mass-density varying inversely as the scaling raised to 1.2 , has got reduced to $8 \times 10^{-27} \mathrm{~kg} / \mathrm{m}^{3}$ and speed to $67 \mathrm{~km} / \mathrm{s} / \mathrm{Mpc}$ and the size of pressure to $43.2 \times 10^{-11} \mathrm{~Pa}$; dark energy-density along with the density of matter and radiation is gradually coming down but is strong enough to be with us till the end of time as evident from the decreasing magnitude and sign of cosmic pressure and source of gravity

and the decaying acceleration since $-\frac{2}{3}<\omega<-\frac{1}{3}$.

If $\omega$ were $-0: 666$, A would have been $1-\frac{1-2}{3-2}=$ and $\mathrm{b}, \frac{2}{H(t)}-t=15.487$ billion years to drive the universe with an acceleration fixed in time; mass density of the universe would have been then varying reciprocally as the scaling.

\section{References}

1. S. Wienberg, Gravitation and Cosmology (Wiley, New York, 1972).

2. M. Charles, K. S. Thorne, and J. A. Wheeler, Gravitation (W. H. Freeman, San Francisco, 
1973).

3. J. V. Narlikar, Introduction to Cosmology (Cambridge University Press, Cambridge, 1993).

4. J. V. Narlikar and T. Padmanabhan, Phys. Rev. D 32, 1928 (1985). https://doi.org/10.1103/PhysRevD.32.1928

5. W. Rindler, Mon. Not. Royal. Astron. Soc. 116, 663 (1986).

6. S. Weinberg, Rev. Mod. Phys. 61, 1 (1989). https://doi.org/10.1103/RevModPhys.61.1

7. A. H. Guth, Phys. Rev. D 23, 347 (1981). https://doi.org/10.1103/PhysRevD.23.347

8. M. Ozer and M. O. Taha, Phys. Lett. B, 171, 363 (1986). https://doi.org/10.1016/0370-2693(86)91421-8

9. W. Chen and Y. Wu, Phys. Rev. D 41, 695 (1990). https://doi.org/10.1103/PhysRevD.41.695

10. A-M. M. Abdel-Rehman, Phys. Rev. D 45, 3497 (1992). https://doi.org/10.1103/PhysRevD.45.3497

11. A. Friedmann, General Rel and Gr. 31, 1991 (1999). https://doi.org/10.1023/A:1026751225741

12. A. A. Penzias and R. W. Wilson, The Astrophys. J. 142, 419 (1965). https://doi.org/10.1086/148307

13. C. L. Bennett, D. Larson, J. L. Weiland, N. Jarosik, G. Hinshaw, N. Odegard, K. M. Smith, R. S. Hill, B. Gold, M. Halpern, E. Komatsu, M. R. Nolta, L. Page, D. N. Spergel, E. Wollack, J. Dunkley, A. Kogut, M. Limon, S. S. Meyer, G. S. Tucker, and E. L. Wright, Astrophys. J. Suppl. Ser. 208, 20 (2013).

14. S. Perlmutter, G. Aldering, G. Goldhaber, R. A. Knop, P. Nugent, P. G. Castro, S. Deustua, S. Fabbro, A. Goobar, D. E. Groom, I. M. Hook, A. G. Kim, M. Y. Kim, J. C. Lee, N. J. Nunes, R. Pain, C. R. Pennypacker, R. Quimby, C. Lidman, R. S. Ellis, M. Irwin, R. G. McMahon, P. Ruiz-Lapuente, N. Walton, B. Schaefer, B. J. Boyle, A. V. Filippenko, T. Matheson, A. S. Fruchter, N. Panagia, H. J. M. Newberg, and W. J. Couch, Astrophys. J. 517, 565 (1999).

15. A. G. Riess, A. V. Filippenko, P. Challis, A. Clocchiatti, A. Diercks, P. M. Garnavich, R. L. Gilliland, C. J. Hogan, S. Jha, R. P. Kirshner, B. Leibundgut, M. M. Phillips, D. Reiss, B. P. Schmidt, R. A. Schommer, R. C. Smith, J. Spyromilio, C. Stubbs, N. B. Suntzeff, and J. Tonry Astron. J. 116, 1009 (1998).

16. I. Zlatev, L. Wang, and P. J. Steinhardt, Phys. Rev. Lett. 82, 896 (1999). https://doi.org/10.1103/PhysRevLett.82.896

17. J. -F. P. Sanchez, Mod. Phys. Lett. A 14, 1539 (1999). https://doi.org/10.1142/S0217732399001632

18. M. V. John and K. B. Joseph, Class. Quantum Grav. 14, 1115 (1997). https://doi.org/10.1088/0264-9381/14/5/016

19. A. S. Al-Rawaf, Mod. Phys. Lett. A 13, 429 (1998). https://doi.org/10.1142/S0217732398000498

20. L. M. Krauss, The Astrophys. J. 501, 461 (1998). https://doi.org/10.1086/305846

21. M. Dewri, J. Sci. Res. 12, 251 (2020). https://doi.org/10.3329/jsr.v12i3.43313

22. S. R. Bhoyar, V. R. Chirde, and S. H. Shekh, J. Sci. Res. 11, 249 (2019). https://doi.org/10.3329/jsr.v11i3.39220

23. Planck 2015 results arxiv.org/abs/1502.015890 astro-ph(0674450).

24. P. M. Garnavich, S. Jha, P. Challis, A. Clocchiatti, A. Diercks, A. V. Filippenko, R. L. Gilliland, C. J. Hogan, R. P. Kirshner, B. Leibundgut, M. M. Phillips, D. Reiss, A. G. Riess, B. P. Schmidt, R. A. Schommer, R. C. Smith, J. Spyromilio, C. Stubbs, N. B. Suntzeff, J. Tonry, S. M. Carroll, The Astrophys. J. 509, 74 (2011).

25. F. Melia, Mon. Not. R. Astron. Soc. 382, 1917 (2007). https://doi.org/10.1111/j.1365-2966.2007.12499.x

26. M. V. John, Mon. Not. R. Astron. Soc. 484, L35 (2019). https://doi.org/10.1093/mnrasl/sly243

27. M. V. John and K. B. Joseph, Phys. Rev. D, 61, ID 087304 (2000).

28. O. Farooq, F. R. Madiyar, S. Crandall, and B. Ratra, Astrophys. J. 835, 26 (2017). https://doi.org/10.3847/1538-4357/835/1/26 